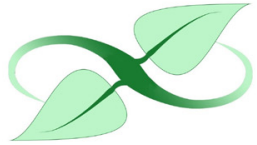

Check for updates

\title{
Growing NHPs for the Future: The 16th Annual Natural Health Products Research Conference and Tradeshow
}

\author{
Paula N. Brown (D), PHD ${ }^{*}, 1,2$, Roy M. Golsteyn $\left(\mathbb{D}, \mathrm{PHD}^{1,3}\right.$ \\ ${ }^{1}$ Natural Health Products Research Society of Canada, Burnaby, BC, Canada \\ ${ }^{2}$ Centre for Applied Research \& Innovation, British Columbia Institute of Technology, Burnaby, BC, Canada \\ ${ }^{3}$ Natural Product and Cancer Cell Laboratories, University of Lethbridge, Lethbridge, AB, Canada \\ *@ paula_brown@bcit.ca
}

\begin{abstract}
The 16th Annual Natural Health Products Research Conference and Tradeshow hosted by the NHP Research Society of Canada (NHPRS) will be held from May 26-29, 2019 in Edmonton, Alberta. Founded in 2003 by a collaboration of academic, industry, and government researchers from across Canada, the NHPRS is a Canadian federally incorporated non-profit organization. The goals of the NHPRS are; (a) to promote scientifically rigorous research and education on natural health products (NHPs), (b) to develop a national research community that encompasses academic, health professional, government and a broadbase of industry stakeholders, (c) to support national research priorities that best enable the informed and appropriate use of NHPs that are safe and efficacious, (d) to increase the capacity for NHP research and education, (e) to facilitate effective NHP knowledge transfer and translation, (f) to support the use of science-based product quality standards and the use of well-characterized materials and protocols in research and (g) to foster value-chain development through interdisciplinary NHP research collaborations and networking. To forward these objectives, the NHPRS has held annual research conferences since inception with themes that highlight the important trends in the ever growing and changing field of NHP Research. The theme of the 16th Annual Conference is "Growing NHPs for the Future". The abstracts are ordered alphabetically by presenting author last name, oral presentations followed by poster presentations. For more information, please visit: http://www.nhprs.ca/.
\end{abstract}

KEYWORDS: NHP Research Society of Canada, NHPRS, Natural Health Products, Research

\section{Acknowledgements}

On behalf of the NHP Research Society of Canada we gratefully acknowledge the support of our conference sponsors and the contributions of the Program Committee, Local Organizing Committee, NHPRS Board of Directors and student volunteers. In particular we would like to acknowledge and thank Dr. Marleny D. A. Saldaña from the University of Alberta who graciously took on the role of Conference Chair for our meeting in Edmonton this year.

Note: Corrections to a few abstract author names were made after the original version was published on May 26, 2019, however, all content remains unchanged. We regret any inconvenience caused. 


\section{Oral Abstracts}

\section{Biologically Active Natural Products: Discovery, Sar, and Interactions with Protein Targets}

\section{Raymond J. Andersen}

Department of Chemistry, University of British Columbia, Vancouver, BC, Canada

The secondary metabolites found in living organisms represent an extremely rich source of novel chemical diversity for academic drug discovery and chemical biology programs. Our group at UBC has amassed a sizable library of crude extracts from marine invertebrates, cultured marine and terrestrial microorganisms, and terrestrial plants. In collaboration with biologists, this crude extract library has been screened for activity in cell-based and pure enzyme assays designed to identify promising natural product lead compounds for the development of drugs. Bioassay-guided fractionation of crude extracts and extensive spectroscopic analysis has been used to identify the structures of pure natural products active in the assays. Biology-oriented chemical synthesis has been undertaken to probe the SAR (structure-activity relationships) for new natural product pharmacophores that we have discovered and to provide material for in vivo testing in animal models. We have used Click chemistry probes and protein $\mathrm{x}$-ray diffraction analysis to study the interactions of bioactive natural products with their molecular targets. Several new drug candidates for the treatment of cancer, inflammation, type II diabetes, and infectious diseases have emerged from this research program. Four of them have progressed to phase II/III clinical trials in humans and others are in preclinical evaluation/development. The lecture will present some recent highlights from our academic natural products drug discovery and chemical biology research.

\section{Product Innovation and the Proposed Self-Care Regulatory Framework Matthew Bown \\ Health Products and Food Branch Inspectorate, Health Canada, Ottawa, ON, Canada}

The Self-Care Framework goals include the creation of consistent and predictable regulatory pathways for all NHP, with less repeatition and the ability to leverage international decisions, for NHPs with higher uncertainty or potentially more risk.

This is intended to be done under several banners:

Quicker Authorizations of Pre-approved Conditions

- Under the SCF, the timelines for products that have already been assessed as safe, including ingredients, combinations of ingredients and various established conditions of use, could be significantly less than the present standards.

Exploring a Period of Time Before Creating Pre-Cleared Information

- Over the past several years, there have been many policies that could lead to the leveraging of previous decisions, cross-referencing decisions and pre-cleared information being created almost immediately after, or even concurrent.

Standards of Evidence

- A low risk product is a low risk product, regardless of whether it is a homeopathic, traditional or modern product. The standards of evidence will be revisited to create a simplified pathway for low-risk (Category I and II) products, akin to but an expansion of the current pathway for licensing documents.

New Technology

- As referenced above, a new system is being built and designed to replace SAS, the EPLA, LNHPD and NHPID that all work together to validate against pre-cleared information.

More PCI

- The proposal is to evolve to a system founded on masses of pre-cleared information.

Automated Processing

- Health Canada is working on an automated process whereby all previous decisions have been transferred from documents to digital data. As such, the ability to "pick-and-play" within the system will be available. 


\title{
O03 Novel Protein-Lipid Composite Nanoparticles as Delivery Systems of Hydrophilic Nutraceutical Compounds \\ Lingyun Chen, Guangyu Liu \\ Department of Agricultural, Food and Nutritional Science, University of Alberta, Edmonton, AB, Canada
}

Food protein and lipid based nanoparticles have attracted recent interest as a means of delivering nutraceuticals. Nanoparticle encapsulation of nutraceuticals faces challenges to overcome for it to be readily applied in the food industry, such as low encapsulation efficiency for hydrophilic compounds and poor stability once in gastrointestinal tract. This research introduces a new protein-lipid composite nanoparticle with an inner aqueous compartment to load hydrophilic nutraceuticals. The protein layer serving as a "scaffold" to which the phospholipid layers are attached. The lipid layer stabilizes the inner aqueous compartment and separate it from the outer water phase, creating greater encapsulation efficiency for the contained hydrophilic compounds (69\% for vitamin B12). In addition, the protein shell could resist a simulated low $\mathrm{pH}$, pepsin gastric environment and then subsequently was able to control the rate of vitamin B12 released into a simulated intestinal environment. An in vitro cell evaluation demonstrated the nanoparticles are internalized into Caco-2 cells via energy-dependent endocytosis to significantly increase the uptake and transport efficiency of vitamin B12. In an in vivo therapeutic study, vitamin B12 loaded nanoparticle increased serum vitamin B12 and reduced the methylmalonic acid more efficiently than the free forms in rats. A 14-day in vivo toxicity study showed no evidence of toxicity to rats.

\section{Is There a Role for Bioactive Oils in the Treatment of Breast Cancer}

\author{
C. J. Field', M. Newell', L. Postovit ${ }^{2}$, V. Mazurak' \\ ${ }^{1}$ Department of Agricultural, Food and Nutritional Science, University of Alberta, Edmonton, AB, Canada \\ ${ }^{2}$ Department of Oncology, University of Alberta, Edmonton, $A B$, Canada
}

Breast cancer continues to be the most frequently diagnosed cancer in women with one in eight women receiving this diagnosis in Canada each year. Although screening and treatment have extended the lives of many women, of those diagnosed, one in twenty-nine will die from the disease. Clearly, there is need for better prevention and treatment strategies. Triple negative breast cancer (ER-PR-HER2-), represents 15-20\% of all breast cancer diagnoses and has the less favourable prognosis. Our work is focused on omega-3 fatty acids on this form of cancer. Our studies using two long chain polyunsaturated omega-3 fatty acids, stearidonic acid [C18:4(n-3)] and docosahexaenoic acid [DHA, (C22:6(n-3)], have demonstrated that both of these fatty acids are cytotoxic to human breast cancer cells both in vitro and when implanted into mice but are not toxic to non-cancer human breast cells. For these experiments, the stearidonic acid came from a modified flax oil and the DHA from either an algae source or a modified Canola oil. Omega-3 fatty acids elicit their anti-cancer effects by acting through multiple pathways including proliferation and apoptosis. Women diagnosed with breast cancer will undergo chemotherapy. Our most recent studies, in the gold standard pre-clinical animal model of triple negative breast cancer, have demonstrated that feeding a diet enriched in DHA improves the action of a first line chemotherapy agent. This work has propelled a phase II randomized clinical trial that will begin this year. (Support from CIHR and Alberta Canola, bioactive oil provided by Bioactive Oils Group UofA, DSM and NuSeed.)

\section{O05 Exploring Panax Ginseng as a Modulator of Epigenetics Signaling in Augmenting Negative and Depressive Symptoms in Treatment Resistant Schizophrenia: Preliminary Results of Multi-site RCT Study \\ Allison Foskett ${ }^{1}$, Simon S. Chiu ${ }^{2}$, Zack Cernovsky², Robbie Campbell ${ }^{2}$, Ed Lui ${ }^{3}$, John Copen ${ }^{4}$, Mariwan Husni ${ }^{5}$, Amanda Singh ${ }^{6}$ \\ ${ }^{1}$ Red Tree Psychological services, Edmonton Alberta; (affiliated), Department of Education Psychology, University of Alberta, Edmonton, AB, Canada ${ }^{2}$ Department of Psychiatry, Schulich School of Medicine, University of Western Ontario, Lawson Health Research Institute, London, ON, Canada ${ }^{3}$ Department of Pharmacology, Schulich School of Medicine, University of Western Ontario, London, ON, Canada \\ ${ }^{4}$ University of British Columbia, University of Victoria Extended Medical Campus, Victoria, BC, Canada \\ 5 Imperial College London, Faculty of Medicine, London, United Kingdom \\ ${ }^{6}$ Queen's University School of Medicine, Kingston, ON, Canada}

Emerging evidence suggests that epigenetics regulating NMDA(N-methyl-D-aspartate)-glutamate signaling may play pivotal role in treatment resistant schizophrenia (TRS). As a putative histone deacetylase (HDAC) inhibitor, Panax 
Ginseng in interacting with glycine- and polyamine-subunit of NMDA receptor complex may augment symptoms of schizophrenia in TRS. The objective of our RCT study was to examine the efficacy and safety of Panax Ginseng (PG) in TRS clients with persistent negative and subsyndromal depressive symptoms and cognitive impairment. We define the therapeutic endpoint as the response rate of $>/=30 \%$ reduction in negative symptoms: SANS scores and the co-primary endpoint as $>/=30 \%$ reduction in depressive symptoms: HAM-D scores. In our multi-site 8-week randomized placebo controlled trial, we recruited schizophrenia patients (SCS) with SANS > 24. Standardized Panax Ginseng (PG): GINSANA-115 (Boehringer-Ingelheim-Pharmaton Switzerland) was administered to 100 mg-group, 200 mg-group and placebo group. We administered Neuro-cognitive Screening (NCS), Positive and Negative Syndrome Scale: PANSS and SANS and HAM-D at baseline and at regular intervals. SCS subjects were maintained on the same dosages of antipsychotics throughout the study. Completer analysis for 43 subjects showed negative symptom response rate for 200-mg PG was 50\% vs $9.1 \%$ for the placebo group, $\mathrm{p}<0.03$. The $200-\mathrm{mg}$ PG group had depressive response rate of $70 \%$, compared with $18.2 \%$ placebo response rate $(\mathrm{P}<0.001)$, No cognitive changes were found for both $\mathrm{PG}$ group. 100-mg PG had no effects. PG was highly tolerated with no serious adverse events. The promising findings of PG in TRS warrant further RCT trial and offer novel therapeutic avenues in schizophrenia (funded by SMRI USA).

\section{O06 Could Nutrigenomics and Epigenetics Underlie the "Gift" of Traditional Practitioners Using NHPs?}

Pierre S. Haddad ${ }^{1,2}$, Charlotte Segala ${ }^{1}$, Noel Raynal ${ }^{1,3}$

${ }^{1}$ Département de Pharmacologie et Physiologie, University of Montréal, Montreal, QC, Canada ${ }^{2}$ CIHR Team in Aboriginal Antidiabetic Medicines, University of Montréal, Montreal, QC, Canada

${ }^{3}$ Research Centrer, Ste-Justine Childrens Hospital, Montreal, QC, Canada

The Traditional Knowledge of traditional practitioners such as Indigenous Elders, herbalists, Traditional Chinese doctors and others is often perceived as empirical knowledge; meaning that it is based primarily on experience. However, many traditional practitioners contend that traditional knowledge is indeed acquired, but also gifted. The basis of such "gifts" has not attracted as much scientific attention as the phytochemical composition and pharmacological activity of the NHPs used by these practitioners. However, new developments in nutrigenomics and epigenetics demonstrate how environmental compounds, such as micronutrients present in traditional NHPs (e.g. vitamins, polyphenols) are differently perceived and handled by an individual's receptors, transporters and enzymes. These include taste receptors (e.g. sweet and bitter) and xenobiotic sensors such as the Constitutive Androstane Receptor (CAR), Pregnane X Receptor (PXR) and Aryl Hydrocarbon Receptor (AHR) that impact on the expression of drug/xenobiotic-metabolizing enzymes such as members of the cytochrome P450 family. Moreover, genes of such taste receptor, xenobiotic sensor and enzymatic proteins are found more and more to be subject to epigenetic modulation in response to similar environmental NHP-derived micronutrients. The communication will explore the possibility that nutrigenomic polymorphisms and epigenetic sensitivity could provide a physiological basis for how certain gifted individuals can better perceive and/or respond to NHP-derived micronutrients.

\section{O07 Recent Advances in DNA Authentication of Herbal Supplements}

\section{Natalia V. Ivanova}

Centre for Biodiversity Genomics, Biodiversity Institute of Ontario, University of Guelph, Guelph, ON, Canada

The DNA authentication of herbal supplements has raised many questions in the NHP industry since its first implementation attempts using Sanger sequencing. Although authentication of plants using DNA remains problematic due to low sequence divergence in many plant genera, requiring analysis of multiple loci, recent technological advances have paved the road towards the customized approach, which can be used for DNA authentication of herbal materials. The HTS sequencing of full chloroplast and whole genomes enables identification of SNPs which can be used to identify loci capable to provide species-level identification and create nucleotide signatures facilitating development of PCR-based or LAMP systems for the authentication. The sensitivity of metabarcoding enables simultaneous detection of source plant DNA along with possible contaminants. Therefore, results should be interpreted considering biocomplexity of living plant coexisting with fungal endophytes and mycorrhizal fungi, as well as the manufacturing process, which can potentially introduce additional DNA sources. The PCR-free approaches such as genome skimming coupled with full genome reference databases offer unbiased quantitative authentication of herbal mixtures. In the past 3 years an increasing number of studies have utilized metabarcoding or SMRT-sequencing of herbal 
supplements coupled with 'gold-standard' chemical analyses using MS-HPLC or TLC. These studies demonstrated increasing evidence that recent advances in HTS sequencing can be utilized by the manufacturers to ensure safety and quality of raw materials and final products.

\section{Zapping Natural Products - How Microwave Extraction Can Improve Extraction Efficiencies}

\section{Steve C. Jakeway}

Radient Technologies Inc., Edmonton, AB, Canada

There was limited innovation in the extraction of natural products until recently. A small number of new techniques - including microwave extraction - have been developed but have been primarily limited to the laboratory. However, Radient has industrialized microwave extraction and can process up to $200 \mathrm{~kg} / \mathrm{h}$ of biomass through a continuous flow process. Radient is building a global footprint using this technology allowing the company to access the benefits of microwave extraction - increased yield, increased purity, reduced solvent, reduced time, and a reduced carbon foot print. A brief background in the theory of microwave extraction will be discussed followed by several examples demonstrating the benefits of this technology over conventional liquid solid extraction.

\section{O09 Canada's GACP Program Meets EU and Health Standards for Cannabis Connie Kehler \\ Canadian Herb, Spice and Natural Health Product Coalition, Saskatoon, SK, Canada}

Growing and selling Cannabis in Canada means meeting Health Canada regulatory requirements. Health Canada regulates the finished product but requires proof of due diligence throughout the whole chain. The EU (European Union) is requiring the same. So how do Cannabis companies prove they have that due diligence? In Canada as in the EU industry has in place Good Agriculture and Collection Practices. In Canada, CHSNC (Herb, Specialty Agriculture and Natural Health Product Coalition) and HSSA (Herb, Spice and Specialty Agriculture Association) have worked with Health Canada, Agriculture Canada and the Canadian Food Inspection Agency to develop internationally recognized auditable certified GACP standards for Specialty Agriculture including Cannabis. These protocols meld seamlessly with existing or pending Good Production Practices and Good Manufacturing Practices up the chain. Canada's GACPs are recognized as a CFIA On farm food safety program and are revalidate with CFIA every 18 months. The program covers production from field and greenhouse to finished product - assuring that quality assurance, product safety and traceability are in place - essential for recalls and compliance. Canada's GACPs have been benchmarked against the EU GACPs and exceed their standards, have mutual recognition with the United States GACPs (American Herbal Products Association) and were built with the support of the World Health Organization. Whether you are a researcher, product developer, QA manager or product manufacturer, this presentation will provide tools and walk you through the steps on how Canada's GACP help you with EU and Health Canada compliance.

\section{Analgesic Activity for Peripheral Pain by Echinacea Extracts is Partially Mediated through the Endocannabinoid System \\ Rui Liu', Nadia Caram Salas², John T. Arnason'1, Cory S. Harris ${ }^{1}$ \\ ${ }^{1}$ Department of Biology, University of Ottawa, Ottawa, ON, Canada \\ ${ }^{2}$ Centro de Investigación Científica y de Educación Superior, Ensenada Baja California, Mexico}

Echinacea alkylamides (AKAs) are reported to have anti-inflammatory and immunomodulatory activities and can be useful to treat related symptoms. AKAs were hypothesized to interact with the endocannabinoid system (ECS) to mediate some of these pharmacological effects. Our research demonstrated in vitro effects on Fatty Acid Amide Hydrolase (FAAH), CB1 and CB2 receptor binding assays. In vivo, both E. angustifolia and E. purpurea root extract elicited dose dependent analygesic activity in a rat paw model of inflammation and pain. Further tests with $\mathrm{CB}$ receptor antagonists AM251 and AM680 co-administrated with Echinacea extract demonstrated a significant reduction of the pharmacological activity. This evidence suggests analgesic activity for peripheral pain was at least partially mediated through the ECS. The reduction of peripheral pain in an animal model by Echinacea may lead to new NHP applications such as relief of arthritis symptoms. 


\title{
011 Rethinking Our Science: Blackfoot Metaphysics Waiting in the Wings
}

\section{Leroy Little Bear}

Officer of the Order of Canada, University of Lethbridge, Lethbridge, AB, Canada

All societies, at one time or another and however they come into being, lay claim to a territory. Within that territory a culture arises from the mutual relationship with the land and everything on it and above it, including the cosmos. A culture consists of paradigmatic concepts, values, and customs. Paradigms are the tacit infrastructures members of the society use for their beliefs, behavior, and relationships. These beliefs, behavior, and relationship norms manifest as the metaphysics of the society. The metaphysics become the interpretive tool to explain everything from science to human affairs. The metaphysics of the modern western world, though not new, largely coalesced during the Enlightenment Era. In science, rationalism, measurement, matter, and mathematics became the basis of the scientific method. Anything that could not be measured was not scientific. But there are other sciences arising out of different metaphysics. The presentation will discuss Blackfoot metaphysics as a basis for a scientific method which may lead to 'opening new doors in fields such as natural health products'.

\section{Flower Development in Medicinal Plants Soheil S. Mahmoud \\ Department of Biology, The University of British Columbia, Kelowna, BC, Canada}

Flowers are the main sites for the biosynthesis and accumulation of numerous important natural products. Among these are monoterpenes (e.g. linalool, myrcene). My lab investigates the roles of genes that control floral development, and those responsible for the biosynthesis of natural products in medicinal plants, using Lavandula angustifolia (lavender) as a model plant. Flower development in angiosperms follows the ABCDE model, which describes the roles of a number of homeotic genes in flower initiation and organ identity. We have identified a group of homeotic transcription factors that control flower development in lavender. We have also isolated a number of transcription factors that control the expression of terpene synthase genes responsible for the production of monoterpenes in higher plants.

\section{Complementary and Integrative Medicine in Paediatric ADHD: Clinical and Pre-clinical Considerations}

\author{
Hajra Mazhar ${ }^{1,2}$, Philippe Robaey ${ }^{1}$, Brian Foster ${ }^{3}$, Candace Necyk ${ }^{4}$, Lyric Oblin-Moses ${ }^{5}$, Cory S. Harris ${ }^{2,6}$ \\ ${ }^{1}$ Children's Hospital of Eastern Ontario Research Institute, Ottawa, ON, Canada \\ ${ }^{2}$ Department of Biology, Faculty of Science, University of Ottawa, Ottawa, ON, Canada \\ ${ }^{3}$ Department of Cellular and Molecular Medicine, Faculty of Medicine, University of Ottawa, Ottawa, ON, Canada \\ ${ }^{4}$ Faculty of Pharmacy and Pharmaceutical Sciences, University of Alberta, Edmonton, AB, Canada \\ ${ }^{5}$ Department of Chemistry and Biomolecular Sciences, University of Ottawa, ON, Canada \\ ${ }^{6}$ School of Epidemiology and Public Health, University of Ottawa, Ottawa, ON, Canada
}

Complementary and integrative medicines (CIM) are commonly used, alone or in combination with prescription medication, among paediatric patients with developmental and neurological disorders but studies focusing on Attention-Deficit Hyperactivity Disorder (ADHD) are sparse. To gain insight specific to CIT and ADHD, we integrated clinical and pre-clinical approaches investigate patterns of use, perceived effectiveness, facilitators and obstacles of use, and potential safety risks. Patient family surveys completed at the Children's Hospital of Eastern Ontario $(\mathrm{N}=71)$ reveal that $44 \%$ of patients have used some form of CIM and $22 \%$ have used herbal medicine. The most commonly reported treatments include nutrient and dietary supplements, elimination diet, homeopathy, physical therapy, echinacea, melatonin, and evening primrose oil. Whereas few $(>10 \%)$ participants agreed to feeling knowledgeable about CAM, approximately one third reported never discussing CIM use with healthcare practitioners and two-thirds agreed to being comfortable discussing the topic. Risks of potential negative side effects, a frequently reported (28\%) reason for not using CIM, adverse event reports involving herbal medicines and ADHD drugs were identified from the FDAble database then assessed for completeness and causality using a suite of validated assessment tools. Most of the identified AERs (18/23) involved three or more substances with inadequate detail to assess multiple potential interactions. Five reports involving only one NHP and one ADHD drug were evaluated for causality with one probable and one possible herb-drug interaction - both involving formulations of methylphenidate and St. John's wort. To further assess potential risk of herb-drug interaction, extracts of popular herbal medicines were tested in vitro for inhibitory effects on the major metabolic enzymes responsible for eliminating most ADHD drugs. 
Herbal extracts exhibited negligible to potent inhibition of carboxylesterase-1 (CES-1), CYP2D6 and CYP3A4 with considerable variability between different products of the same species. Collectively, these results demonstrate that use of CIT by paediatric ADHD patients is widespread yet often undisclosed to medical practitioners. While clinical and pre-clinical evidence of risk appears low, open communication with doctors and continued research would help avoid herb-drug interactions and support patient health.

\title{
014 Processing Innovations on NHPs - Sustainable Production of Bio-actives Using the Mini-biorefinery Concept
}

\section{Angela A. Meireles}

University of Campinas, Sao Paulo, Brazil

Nowadays, the increased demand for technologies that are harmless to the human being and protective of the environment is provoking changes in the agro based industries. This brought the opportunity of translating the biorefinery concept of fuel-based industries to smaller and frequently multitask processing factories such as for instance the case of production of extracts from condimentary, medicinal or spice processing industries. These factories produce enormous amounts of residue or byproducts; up-to-few years back they were discarded frequently considering only the regulatory demands of local authorities. Instead of considering the production of a target compound from a given biological matrix the problem can be solved using the integration and intensification of processes translating the biorefinery concept to a mini-biorefinery for sustainable production. For instance, from turmeric the target component is the mixture of curcuminoids. Nonetheless, turmeric volatile (essential) oil is a mixture of terpenes rich in turmerones that find various medical applications. Additionally, the unflavored and depigmented solid residue is a source of a flour rich in proteins, starch, and fibers. Thus, this lecture will focus on the use of process integration to obtain several products from biological matrices.

\section{Phytochemical Comparisons of Oplopanax Horridus (Devil's Club) to Guide Sustainable Harvesting}

\author{
Hazrah Moothoo', Paula N. Brown', Allison R. McCutcheon², Roy Upton ${ }^{3}$ \\ ${ }^{1}$ British Columbia Institute of Technology, Burnaby, BC, Canada \\ ${ }^{2}$ Independent Researcher, Vancouver, BC, Canada \\ ${ }^{3}$ American Herbal Pharmacopoeia, Scotts Valley, CA, United States
}

Devil's club (Oplopanax horridus (Sm.) Miq.) is a well-known deciduous shrub with a distribution range in Western North America from Alaska through British Columbia and down south to Oregon and east to Idaho and Montana. Stands of devil's club are often found along seepage sites and streams within forest habitats. The plants are highly valued spiritually, medicinally and considered sacred by the First Nations. Ethnobotanical surveys and anecdotal accounts have noted the preparation and application of different plant parts to target specific symptoms relating to coughs, colds, aches and pains. Samples were collected throughout Alaska, Washington and British Columbia and plant parts such as whole stem, inner/outer stem bark and rhizome were dried and analyzed using high performance liquid chromatography. Vouchered specimens were included as part of the collection. Preliminary results show that the phytochemical profiles of the inner bark of horizontal stem displayed higher amounts of 8 characteristic compounds across the geographical range compared with plant parts derived from the vertical stem. The preliminary data supports the differential medicinal use of select plant parts. Determining the most appropriate plant parts to sustainably harvest will support conservation efforts. Additional specimens should be collected throughout the seasons over the geographical region to strengthen the statistical power of the analysis.

\section{Metabolomics-Guided Food Ingredient Discovery}

\section{Susan J. Murch}

Department of Chemistry, University of British Columbia, Kelowna, BC, Canada

Each piece of a plant contains somewhere between 30,000-55,000 distinct chemicals. An average cup of coffee has about 8,400 distinct chemicals while a cranberry has about 28,000 and a glass of wine has about 8,600 chemicals. For food plants, between 700-1,100 phytochemicals have been fully characterized and we estimate that an average person's diet contains at least 200,000 unknown plant chemicals per day. Some of these chemicals are beneficial to health such as 
antioxidants, antimicrobials, pre- and pro-biotics, immune stimulators, anxiolytics, adaptogens and so on. Other naturally occurring plant chemicals are neurotoxins, hepatotoxins, carcinogens and other poisons. Traditional methods for discovery and description of the chemical constituents of food required a complex process of extraction, solvent partitioning, fractionation, isolation, purification and structure elucidation. We have developed a metabolomics-guided method of phytochemical discovery (MGPD) with statistical tools to eliminate false discoveries and identify food ingredients as well as metabolite families, pathways and relationships. Applications of logical algorithms can be used to identify novel chemicals in complex mixtures through metabolite relationships, predicted enzymatic, redox or spontaneous reactions and metabolite clustering. Using these approaches, we have discovered new potential high-vale food ingredients from and medicinally active phytochemicals in Neurolaena lobata, Scutellaria, Hypericum Artocarpus, Vaccinium and Cannabis.

\title{
017 Beneficial Effects of Functional Peptides from Food Proteins
}

\section{Traj Nibber'1, Pamela Ovadje', Shanna Banman², Robert Ippolito²}

${ }^{1}$ Advanced Orthomolecular Research, Calgary, $A B$, Canada

${ }^{2}$ Agri-Food Discovery Place, University of Alberta, Edmonton, $A B$, Canada

The natural health industry and consumers are rapidly moving towards the development of functional foods, which are generally foods that have potentially positive health effects, beyond meeting the basic nutritional needs. The potential for the reduction of disease risk is expected to be higher when functional foods are introduced into diet and health planning. Studies have shown that there are bioactive peptides within the primary structures of food that remain inactive until they can be released and activated during enzymatic hydrolysis, promoting their health benefits. Advanced orthomolecular Research has been researching the development of a protocol, suitable for the commercial scale up production of pea protein hydrolysate that retains its cardiovascular benefits. Peptides from pea have the ability to modulate the renin-angiotensin system (RAS) by decreasing the activities of renin or angiotensinconverting enzyme (ACE), thereby regulating blood pressure. In-vitro and in-vivo enzymatic hydrolysis of pea protein suggests a significant inhibitory activity of pea protein hydrolysate $(\mathrm{PPH})$ on both renin and ACE inhibitory activity. The scale-up process, however, is lacking in being able to maintain the ACE and/or renin inhibitory activity of PPH. The focus of this research is to establish a protocol for environmentally sustainable development of a commercial scale $\mathrm{PPH}$ that retains the cardiovascular supporting attributes that have been identified in bench scale extractions. The results of this study will enhance our understanding of functional foods, and the best way to exploit their characteristics for the development of a commercial product or health benefits.

\section{Case Study in Identification of Adulterants in Herbal Products Through Multi-disciplinary Methods}

\author{
Rob O'Brien', Sean Mackenrot'1, Sheena Kuo', Fabrice Berrue ${ }^{2}$ \\ IISURA, Burnaby, BC, Canada \\ ${ }^{2} N R C$, Halifax, NS, Canada
}

Many take herbal products because they feel more comfortable self-medicating with something that is "natural". However, so-called "natural" herbal remedies are sometimes adulterated with synthetic substances. In the past, these adulterants are easy to detect and identify with mass spectrometry. Compounds are now being synthesized to escape direct mass spectrometry identification. This presentation will discuss a case study with a herbal supplement where additional chemical and biochemical techniques are deployed to supplement traditional mass spectrometry data.

\section{The Antibiotic Activities Characterized in Alberta Prairie Plants}

\author{
Megan Puchbauer ${ }^{1}$, Ashtin Halmrast ${ }^{1}$, Chad Beck ${ }^{1,2}$, Sean Sanders ${ }^{1}$, Craig James ${ }^{1}$, Carlee Ayley ${ }^{1}$, \\ Michaela Prozniak', Deserae Tailfeathers ${ }^{1,2}$, Steven MacRae ${ }^{1}$, Roy M. Golsteyn ${ }^{2}$, David Williams ${ }^{3}$, \\ Leanne DuMontier ${ }^{1}$, Raymond Andersen ${ }^{3}$, Sophie Kernéis ${ }^{1}$ \\ ${ }^{1}$ Microbial Research Group, Lethbridge College, Lethbridge, AB, Canada \\ ${ }^{2}$ Natural Product Laboratory, University of Lethbridge, Lethbridge, AB, Canada \\ ${ }^{3}$ Department of Earth, Ocean and Atmospheric Sciences, University of British Columbia, Vancouver, BC, Canada
}

Since 2017, the World Health Organization has declared antibiotic resistance to be a topic of high health priority. They report that if no action is taken we will return to an era when people are at great risk of dying from common infections. One of their recommendations is to discover new antibiotics especially for bacteria from the ESKAPE group; a 
group that are causing more nosocomial infections and for which we have more antibiotic resistant strains. Current antibiotics have been solely isolated from fungi and bacteria; none has a plant origin even though plants have been used for many years in traditional medicine. Our project is to discover antibiotic molecules from plants native to Alberta. We have developed a plant extract library with ninety extracts coming from seventeen plant families. The techniques to test the antibiotic activities of our plant extracts are in place in our laboratory. From twenty extracts screened for their antibiotic activities, eight are active against Staphylococcus aureus, and four are active against Acinetobacter baumanii. The activities were identified in plants from different families in favour of finding different chemicals. We have isolated two chemicals showing antibiotic activities from one extract. This study demonstrates the potential of plants as sources of chemicals with antibiotic properties. This research forms the foundation for serving needs in human and animal medicine, agriculture, and cosmetics.

\section{O20 Rhodiola Rosea - Sustainable Production of an Endangered Adaptogen Nelda Radford}

RR 2, Site 14, Box 16, Olds, $A B$, Canada

Rhodiola rosea has been used throughout time to combat fatigue and stress, and improve cognitive abilities. The herb in its native environments in northern hemisphere colder climates is now considered endangered in most countries. Efforts to cultivate Rhodiola rosea have been attempted in several countries with limited success. A cooperative of farmers in Alberta has developed methods and provides support for growing Rhodiola rosea locally. The climate in portions of Alberta is ideal to develop commercial levels of phytochemicals within a 6-year crop cycle. Challenges to cultivation abound. Native colonies have also been discovered in Labrador and New Brunswick, and efforts to cultivate are beginning in these provinces. Alberta and Canadian GACP and NHP standards are essential to consumer acceptance of this raw material worldwide.

\section{Using a Six Sigma Method to Verify Quality Systems Can Improve Product Outcomes Rebecca Robertson \\ British Columbia Institute of Technology, Burnaby, BC, Canada}

Food and Natural Health Products manufacturers must verify that their quality systems are operating as intended and effective. This is generally achieved by such activities as validating and auditing the system and performing targeted sampling and testing ${ }^{1}$. While these procedures have great value, they do not examine a full process while it is running which means that process nonconformities may go undetected for long periods of time until a problem emerges. This presentation examines use of an adapted six sigma performance metric, defects per opportunity (DPO), to develop a Defect Opportunity Checklist (DOC) to measure and analyze the defects associated with a receiving process. Receiving processes must be verified to ensure raw materials and the related conveyance are being properly assessed for incoming hazards and quality problems, and that new lots of raw materials are being recorded and placed into inventory in a manner that facilitates their traceability through to processing into finished products. In this case study, the manufacturer was having difficulty tracking the lot numbers of their main raw material thus compromising ingredient traceability. The DOC was developed from the company's Receiving Standard Operating Procedures and the defect rate was calculated from two independent runs performed over a month where it was observed that the defect rate was 43 and $49 \%$ respectively. The defects were then categorized and plotted on a Pareto chart while the reasons for the poor lot code tracking were analyzed and illustrated on a Cause \& Effect Diagram. This research is important for the Food and Natural Health Products industries because this is a simple, effective tool that clearly indicates the status of any given process and provides insight into how to correct the identified nonconformities.

1. FAO. The Hazard Analysis and Critical Control Point (HACCP) System. Food Quality and Safety Systems - A Training Manual on Food Hygiene and the Hazard Analysis and Critical Control Point (HACCP) System. Rome : 1998.

\section{O22 Cannabidiol (CBD) in the Regulatory Framework of Self-care Consumer Health Products Adrian Rodriguez \\ NHP Consulting/Cannabis Compliance Inc., Mississauga, ON, Canada}

On October 17, 2018, the Cannabis Act and Cannabis Regulations came into force creating the legal framework for controlling the production, distribution, sale and possession of cannabis across Canada. Prior to this, Health 
Canada had proposed an overlap between the Cannabis Act and the Food and Drugs Act (FDA) for the production and distribution of cannabis-containing consumer health products including natural health products (NHP), pharmaceuticals, cosmetics, and veterinary health products, presenting an incredible opportunity for licensed producers of cannabis, manufacturers of consumer health products, and consumers alike. The act and regulations, however, contradicted this proposal by introducing cannabis and its phytocannabinoids, including cannabidiol (CBD), into the Prescription Drug List (PDL) and Schedule 2 of the Natural Health Products Regulations (NHPR), rendering them prohibited ingredients in consumer health products. CBD is of special interest to the NHP industry given the evidence available to support its safety and efficacy within a self-care model suitable for the regulated framework of NHPs. This presentation will examine the case for a "switch submission" for CBD, an application submitted to Health Canada in support of switching a medicinal ingredient from prescription to non-prescription status. Based on the data requirements set by Health Canada for this type of submission, the regulatory and scientific rationale for allowing CBD in self-care consumer products will be evaluated. Moreover, the current Canadian regulatory framework and requirements to produce $\mathrm{CBD}$ products will be discussed.

\section{Processing with Pressurized Fluid Systems: Natural Health Products}

\section{Marleny D. A. Saldaña}

Department of Agricultural, Food and Nutritional Science, University of Alberta, Edmonton, AB, Canada

The demand of bioactive compounds is increasing as the consumption of these natural based products is associated with different health benefits. Phenolic acids found in various crops have antioxidant and antimicrobial activities and their consumption have been associated with a lower incidence of cancer, heart disease, and diabetes. Research in my laboratory has focused on the use of pressurized fluids to extract these phenolic compounds to be used in various applications. Pressurized fluids, such as subcritical water and supercritical $\mathrm{CO}_{2}\left(\mathrm{SC}_{-} \mathrm{CO}_{2}\right)$ as "green" and environmentally friendly solvents, can be used for the extraction of phenolic compounds from crops as well as for enzymatic reactions involving phenolic acids. Phenolic compounds were extracted from crops using pressurized fluids in stainless steel reactors at different temperatures, pressures and times. Extracts obtained using pressurized fluids were evaluated for their phenolic content and antioxidant activity. Results indicated that the total phenolic content and antioxidant activity of the extract increased with extraction temperature. In addition, a phenolic acid was reacted with triglycerides of flax oil using lipase enzyme in $\mathrm{SC}-\mathrm{CO}_{2}$ media in a laboratory-scale supercritical system at different temperatures ranging from 40 to $80^{\circ} \mathrm{C}$, pressures from 4 to $35 \mathrm{MPa}$ and times of up to $53 \mathrm{~h}$. Results have shown that $\mathrm{SC}-\mathrm{CO}_{2}$ is a promising green solvent for the enzymatic synthesis of phenolic lipids. In addition, selected phenolic compounds were reacted with starch to produce bioactive films. Results have shown that bioactive films can be processed using subcritical water for different applications. Additional examples will be shown for pressurized fluid systems from rhodiola, artemisia and berry pomace.

\section{Cranberry Pomace: A Feedstock for the Recovery of Antioxidants Using Sub/Supercritical Fluids

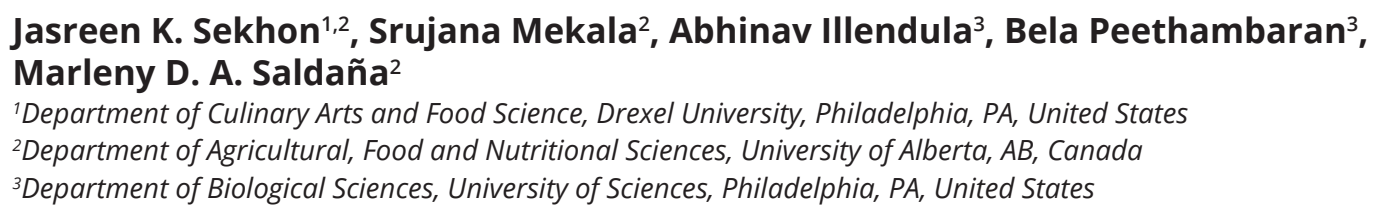

Cranberries contain one of the highest levels of flavonoids of most commonly consumed fruits. The purpose of this study was: (a) to recover polyphenols from cranberry pomace $(\mathrm{CP})$ using sequential extraction with pressurized fluids ( $30 \%$ ethanol and/ or $100 \%$ ethanol at 50 bar; temperatures of $60-160^{\circ} \mathrm{C}$ ), (b) demonstrate health benefits within an in vitro breast cancer model (MDA-MB-231 cell line) using MTT assay, and (c) evaluate polyphenol stability of the freeze dried or supercritical CO2 dried extract. Maximum anthocyanin content (4.6 \pm 0.1 $\mathrm{mg} \mathrm{Cy} 3 \mathrm{Gu}$ ) was recovered from dried $\mathrm{CP}$ powder extracted with $100 \%$ ethanol at $80^{\circ} \mathrm{C} / 50 \mathrm{bar}$, and maximum total phenolic content $(36.5 \pm 5.5 \mathrm{mg}$ GAE/g dried CP) was observed when CP was extracted with $30 \%$ ethanol at $160^{\circ} \mathrm{C} / 50$ bar. Sequential extraction with $100 \%$ ethanol (for $15 \mathrm{~min}$ ) followed by $30 \%$ ethanol (for $15 \mathrm{~min}$ ) at $140^{\circ} \mathrm{C}$ resulted in high anthocyanin content $(\sim 3.5 \pm 0.65 \mathrm{mg} \mathrm{Cy} 3 \mathrm{Gu})$ and total phenolic content $(\sim 27 \pm 1.36 \mathrm{mg}$ 
GAE/g dried CP). Higher antioxidant activity values in the extracts were obtained from sequential extraction at $140^{\circ} \mathrm{C}(\sim 1.8 \pm .01 \mathrm{mM}$ Trolox/L extract $)$ compared to the extracted with $100 \%$ ethanol at $80^{\circ} \mathrm{C}(0.7 \pm 0.1 \mathrm{mM}$ Trolox/L extract). This indicates anthocyanins have little contribution to the antioxidant activity compared to other polyphenols extracted at higher temperatures using aqueous ethanol. A significant death in the breast cancer cell line MDA-MB-231 was achieved by incubating cancer cells with extracts recovered from 100\% ethanol extraction or sequential extraction with 30\% ethanol. However, minimum IC50 values were obtained when CP was extracted with $100 \%$ ethanol for $5 \mathrm{~min}$. Results for the stability of antioxidants in an encapsulated powder product will be presented. This study demonstrated proof-of-concept.

\title{
025 Not all Pathways to Licensing are Straightforward: The Story of Thyrodine ${ }^{\circledR}$ Sharan Sidhu \\ Vernon, BC, Canada
}

The Natural and Non-Prescriptions Health Products Directorate currently license products based on three categories. Categories I and II are straightforward and allows products to come to market with relative ease and category III require more comprehensive scientific review. In some cases, additional requisites need to be meet and additional processes must be completed. Thyrodine ${ }^{\circledast}$ is one such product that not only required licensing as per the NHPRs requirements, but also had to provide processing development and validation, testing validation as well as a switch submission. This product is an example of development and innovation overcoming several regulatory constraints.

\section{The Sustainable Production of the Raw Materials for the Natural Health Products}

Jan V. Slama

501-3292 Production Way, Burnaby, BC, Canada

Where do the herbs in your NHP's come from? How are they grown? Some products are made from endangered species whereas others are produced after mass destruction of tropical forests. Ethical standards are needed to make sure that there is a balance between our health needs and our precious environment. What steps can be taken in ensuring sustainability? This presentation will discuss specific examples of efficient production of sustainable NHP's. Most of the herbal products produced by one of Isura's clients (Natural Factors), use raw herbal materials produced by their organic farming operation, as well as by sustainable wildcrafting practices. The raw materials production of their farming operation is carefully managed. It follows the ethical standards, and it includes seed production and preservation programs, land and soil husbandry, biogenic farming practices, and the utilization of invasive species by wildcrafting. These practices, as well as standardized harvesting and processing procedures, assure a long-term sustainable source of the raw materials, and preservation of the potentially endangered species of the medicinal herbs.

\section{Prairie Plants as Sources of Natural Products with Scientific and Medicinal Interest: Identification of a Compound that Changes Nuclear Structure in Human Cancer Cells}

\author{
Jan Tuescher ${ }^{1}$, Chad Beck ${ }^{1}$, Sophie Kernéis², Locke Spencer ${ }^{3}$, Raymond Andersen ${ }^{4}$, Roy M. Golsteyn ${ }^{1}$ \\ ${ }^{1}$ Natural Product Laboratory, University of Lethbridge, Lethbridge, AB, Canada \\ ${ }^{2}$ Microbial Research Group, Lethbridge College, Lethbridge, AB, Canada \\ ${ }^{3}$ Department of Physics and Astronomy, University of Lethbridge, Lethbridge, $A B$, Canada \\ ${ }^{4}$ Department of Earth, Ocean and Atmospheric Sciences, University of British Columbia, Vancouver, BC, Canada
}

Plants within the Prairie ecological zone evolved under the selective pressures of a short growing season and grazing by large herbivores. Under these conditions, some plants produce secondary metabolites that are toxic to mammals, including humans. We have prepared a unique extract library from Prairie plants representing 20 taxonomical families. We use cell-based phenotypic assays to investigate how the extracts or chemicals isolated from these plants affect human cells. Extract PP-630 induced a vacuolated phenotype when applied to seven different cell lines. This phenotype was photoinduced, requiring exposure to light at 405 or $660 \mathrm{~nm}$ at doses of 1000 and $1250 \mathrm{~mJ} / \mathrm{cm}^{2}$, respectively, in which $>90 \%$ of cells would acquire a nuclear vacuole. By the MTT assay at 96 hours, light exposed PP-630 treated 
cells showed a 10 -fold higher toxicity $(\mathrm{EC}=7.8 \mu \mathrm{g} / \mathrm{mL})$ than treated cells without light exposure $(\mathrm{EC}=73 \mu \mathrm{g} / \mathrm{mL})$. The natural product, pheophorbide A, was isolated by biology-guided fractionation from PP-630 and demonstrated to be the source of the vacuole-inducing activities. PP-630-induced and pheophorbide A-induced vacuoles could be distinguished from other reported vacuole-inducing agents, such as cyclosporin A or curcumin. We observed that the nuclear envelope protein lamin B was concentrated at the site of the nuclear vacuole, as shown by immunofluorescence microscopy. Strikingly, nuclear vacuoles have also been observed in cells from patients of the Pelger-Huët anomaly, which is a rare disease linked to mutations in the human lamin B receptor. Our research reveals that Prairie plants are a rich source of natural products for use as tools to investigate cell function and potential medical treatments.

\title{
028 Collection, Cultivation and Chemical Analysis of Osha: Investigating an At-risk Medicinal Plant
}

\author{
Stacy Wise ${ }^{1}$, Hazrah Moothoo ${ }^{2}$, Wendy Applequist ${ }^{3}$, Paula N. Brown ${ }^{2}$ \\ ${ }^{1}$ BearRoots New Mexico, Santa Fe, NM, USA \\ ${ }^{2}$ Centre for Applied Research \& Innovation, BC Institute of Technology, Burnaby, BC, Canada \\ ${ }^{3}$ Missori Botanical Garden, St. Louis, MO, USA
}

Ligusticum porteri Coulter \& Rose, native to the Rocky Mountains of North America, is a revered wildcrafted medicinal plant of the Apiaceae family. Many groups have expressed concern about the health and future of wild osha populations, as it is a slow-growing perennial threatened by over-harvest, livestock grazing, climate change, and habitat loss. We present research into three approaches to wild osha conservation - 1) best harvest practices, 2) chemical analyses to justify substitution with less endangered plants, and 3) attempts at domestication. Recent investigations by the University of Kansas, BC Institute of Technology, Missouri Botanical Garden, and American Herbal Products Association surveyed current osha populations in southern Colorado, and studied how wild stands recover from harvests. The marker compounds of note for Ligusticum in pharmacopoeias, namely vanillin, ferulic acid and Z-ligustilide, were compared across multiple accessions of six different Ligusticum species including the "true osha," L. porteri. Variation within species was nearly as large as the interspecies variation, suggesting that these marker compounds are not suitable for species differentiation, however there may be justification for substitution with the less threatened species for the same indications. Finally, we present summary of cultivation efforts in the western US and Canada, and highlight the difficulties encountered, the many failures, and a rare success.

\section{O29 Food Proteins as a Source of Bioactive Peptides Against Cardiovascular Diseases}

\section{Jianping Wu}

Department of Agricultural, Food and Nutritional Science, Cardiovascular Disease Research Centre, University of Alberta, Edmonton, $A B$, Canada

Food proteins are a major macronutrient. The objective of the presentation was to study the potential of protein derived peptides against hypertension. Three peptides were characterized from egg protein. In vivo antihypertensive activity was studied in spontaneously hypertensive rats (SHRs) using the telemetric method. Second order branches of the mesenteric artery were carefully isolated after animal euthanasia, and analyzed by wire-myography. Effect of peptides on vascular protein expression including adhesion molecules was determined using western blotting. The level of free radicals, reflected in nitrotyrosine, was determined using immunofluroscence. Oral administration of IRW, IQW, and LKP (15 mg/kg BW) significantly lowered mean arterial pressure (MAP) by 40, 19, and 30 $\mathrm{mmHg}$, respectively, over a period of 18 days. IRW treatment led to decreased angiotensin II (Ang II) levels, restored nitric oxide (NO) dependent vasorelaxation, and decreased expression of inflammatory markers such as ICAM-1 and VCAM-1 as well as tissue fibrosis. Therefore, the antihypertensive effect of IRW was likely mediated through ACE inhibition, endothelial nitric oxide synthase, and anti-inflammatory properties. IQW and LKP treatments also reduced plasma Ang II level and restored NO-dependent vasorelaxation; however, only IQW treatment reduced ICAM-1 expression and nitrotyrosine levels in the arteries. The antihypertensive activities of IQW and LKP were likely mediated through similar pathways involving increased NO-mediated vasorelaxation and ACE inhibition, but only IQW reduced vascular inflammation and oxidative stress. Food protein-derived peptides may have great potential as an alternative strategy in the prevention and management of hypertension. 


\section{Poster Abstracts}

\section{P01 Bioactive Lipids and Protein Removal by Fractionation of Barley Bran Azadeh Aghashahi, Marleny D. A. Saldaña \\ Department of Agricultural, Food and Nutritional Science, Faculty of Agricultural, Life and Environmental Science, University of Alberta, Edmonton, $A B$, Canada}

Barley bran obtained from the pearling process is rich in bioactive compounds (beta-glucans, protein and lipids). Beta-glucans, approved by Health Canada help with cholesterol and glucose reduction. Barley proteins are a good source of essential amino acids that can be used as foaming/emulsifying agents in the food industry. Barley lipids are a rich in tocopherols, tocotrienols, phytosterols and alkylresorcinols. The aim of this study was to fractionate barley bran using supercritical $\mathrm{CO}_{2}\left(\mathrm{SC}-\mathrm{CO}_{2}\right)$ to obtain bioactive lipids followed by starch and protein removal to obtain barley bran fiber. Lipid extraction was performed by two methods, including traditional and $\mathrm{SC}-\mathrm{CO}_{2}$ extraction. $\mathrm{CO}_{2}$, which is non-toxic and non-flammable at supercritical conditions $\left(31^{\circ} \mathrm{C} \& 7.4 \mathrm{MPa}\right)$, was used. Furthermore, the crude lipid and defatted meal obtained by $\mathrm{SC}-\mathrm{CO}_{2}$ had no toxic solvent residue. Lipid extraction was carried out at different pressures $\left(200 \& 300\right.$ bar) and temperatures $\left(40 \& 70^{\circ} \mathrm{C}\right)$ for $2 \mathrm{~h}$. After lipid extraction using $\mathrm{SC}-\mathrm{CO}_{2}$, the bran was destarched using enzymes. Protein extraction was investigated using sodium chloride $(1-10 \% \mathrm{w} / \mathrm{v})$, sodium hydroxide $(50 \mathrm{Mm})$, and ethanol (50 and 70\% v/v). Protein content was determined in the residue. Majority of lipids was extracted within the first $30 \mathrm{~min}$ at all conditions investigated, with a maximum recovery of $97 \%$ at $300 \mathrm{bar} / 70^{\circ} \mathrm{C}$ after $2 \mathrm{~h}$ extraction. Enzymatic starch removal was successful to reduce the starch content from 10.91 to $0.91 \%(\mathrm{db})$, while beta-glucan and protein were concentrated in the final destarched bran. Sodium hydroxide solution was the most effective to reduce the protein content from 25 to $12 \%$ in the final residue. Fractions obtained rich in bioactives can be used in the food and nutraceutical industry.

\section{P02 Immune-modulatory Effects of Yemeni Sidr Honey: Implications for Anti-proliferative Effects on Cancer Cells}

\section{Danah Almnayan, Robert Lafrenie}

Health Sciences North Research Institute, Laurentian University, Sudbury, ON, Canada

Honey has become popular as a potential treatment for several ailments, including many cancers. Honeys from different parts of the world have been shown to have different anti-proliferative, immune-modulatory, and anti-inflammatory actions. For example, Yemeni Sidr Honey (YSH) is world-renowned for its anti-inflammatory activity and has been suggested to have anti-cancerous and/or immune-modulatory actions. However, empirical evidence is lacking. We have been investigating YSH's apoptotic, anti-proliferative, and/or immune-modulatory activities in in vitro models of cancer growth and immune cell function. Our studies have shown that YSH is able to inhibit proliferation, and induce apoptosis in several human cancer cells lines. Treatment of breast cancer cell lines (MDA-MB-231 and MCF-7) and cervical cancer cell lines (Hela) with 1\% (v/v) YSH in media almost completely inhibits cell proliferation, and promotes cell apoptosis. In addition, treatment of the THP-1 monocyte-like cell line with YSH can alter macrophage polarization in vitro. Treatment of THP-1-derived macrophages with YSH for 3-5 days, enhances the expression of cell surface markers associated with the pro-inflammatory M1 macrophage subtype, and does not enhance markers associated with the M2 repair subtype suggesting YSH may have an anti-cancer potential. Future, experiments will determine if the rate of YSH-induced apoptosis in human cancer cell lines co-cultured with THP-1-derived macrophages depends on M1/M2 status.

\section{P03 Morphological and Particle Size-based Characterization of Fava Beans as a Tool for Efficient Separation of Grain Components}

\section{Jeganathan Brasathe, Jun Gao, Thava Vasanthan, Feral Temelli}

The University of Alberta, Edmonton, $A B$, Canada

Fava bean (Vicia faba L.) is a protein-rich pulse, which is also a good source of dietary fibre and other bioactive compounds with potential health benefits. However, it is underutilized for human consumption mainly due to some negative impacts of non-nutritional compounds. The overall objective of this study is to develop environmentally friendly approaches for the efficient separation of grain components (protein, dietary fibre, starch and lipids) 
for their eventual use in food applications. As a first step, the morphology of interface between split surface of the two cotyledons (SS) and the external seed coat surface (ES) of fava beans [Snowbird (LT), Athena (HT)] were examined using scanning electron microscope [SEM] and helium ion microscope [HIM]. The SS was protected by a cuticle layer, which showed morphological similarities to the ES, warranting the uniformity of the beans during pearling. Based on the extensive component distribution analyses and SEM and HIM imaging of the pearled fractions, proteins and dietary fibre were more concentrated in the external layers, which gradually reduced towards the inner layers, whereas starch content showed an inverse trend. With the increasing particle size ( $<75 \mu \mathrm{m}$ to $500 \mu \mathrm{m})$, the protein and dietary fibre content increased whereas starch showed an inverse trend. The subsequent wet fractionation resulted in protein isolates ( $>90 \%$ protein) and value-added protein and dietary fibre-rich fractions. These data suggest that inclusion of pearling/air-current assisted particle separation (ACAPS) as upstream processing steps in fava bean fractionation can generate novel food ingredients for subsequent processing.

\title{
P04 Toxicology, Pharmacology and Clinical Reports of a Peruvian Herbal Formulation (A4+) for Treating Liver Disease
}

\author{
José Cabanillas ${ }^{1}$, Francis H. Y. Green ${ }^{2}$, Hugh A. Semple ${ }^{3}$, Brian D. Sloley , Grishma Shrestha ${ }^{5}$, \\ Steven K. H. Aung6 \\ 'Sabell Corporation, Calgary, AB, Canada \\ ${ }^{2}$ Department of Pathology and Laboratory Medicine, Cumming School of Medicine, University of Calgary, Calgary, AB, Canada \\ ${ }^{3}$ Redcliff, $A B$, Canada \\ ${ }^{4}$ Phytovox Inc., Edmonton, $A B$, Canada \\ ${ }^{5}$ Snyder Institute for Chronic Diseases, Cumming School of Medicine, University of Calgary, Calgary, AB, Canada \\ ${ }^{6}$ Dr Steven K H Aung Clinic, Edmonton, AB, Canada
}

A4+ is composed of three plants Cordia lutea, Curcuma longa and Annona muricata. Individually, they have been used traditionally to treat liver diseases in the Peruvian Amazon. Together this combination of herbs in the ratio of 80:10:10 has proven to be synergistic and highly effective in treating various liver diseases including viral hepatitis. A4+ was approved by the Natural Health Product Directorate of Health Canada (license NPN 80033347) in July 2012 as a hepato-protectant. A4+ showed no evidence of toxicity in a 28-day repeated-dose test or in a micronucleus study for cytotoxicity and genotoxicity with a no observed adverse effect level (NOAEL) of $2000 \mathrm{mg} / \mathrm{kg}$. Extracts from the three plants were analyzed using high performance liquid chromatography (HPLC) coupled to diode array absorbance (DAD) and positive mode electrospray mass spectrometry. Each plant had a distinct chemical fingerprint. Using a variety of in vitro and in vivo tests, A4+ showed a reduction in acute inflammation and had potent anti-oxidant, anti-viral and gastric mucosal protective activity. It was protective in an immune mediated hepatitis model and improved behavioral function in animals with late stage liver disease. In two $(n=6 \& n=10)$ exploratory studies of patients with Genotype 1 Hepatitis C, A4+ dramatically improved health related quality of life scores (SF-36 and Beck) and significantly increased prothrombin and serum cholinesterase levels suggesting liver function improvement. In clinical practice, positive results have been observed in individual patients suffering from a variety of liver disorders. A4+ has proved tremendously beneficial in patients with fatigue, infectious diseases, NASH, hepatitis, cirrhosis, immune deficiencies and as an adjunct to chemotherapy.

\section{P05 Egg White Hydrolysate Enhances Insulin Signaling in White Adipose Tissue Despite No Improvement in Local Inflammatory State \\ Stepheny C. de Campos Zani ${ }^{1,2}$, Forough Jahandideh ${ }^{3}$, Jianping $\mathbf{W u}^{3}$, Catherine B. Chan ${ }^{1,2,3}$ \\ ${ }^{1}$ Department of Physiology, Faculty of Medicine and Dentistry, University of Alberta, Edmonton, AB, Canada \\ ${ }^{2}$ Alberta Diabetes Institute, Edmonton, AB, Canada \\ ${ }^{3}$ Department of Agricultural, Food \& Nutritional Science, Faculty of Agricultural Life and Environmental Sciences, University of Alberta, Edmonton, $A B$, Canada}

Type 2 diabetes (T2D) is often associated with obesity and inflammation. Local white adipose tissue (WAT) inflammation is believed to worsen insulin resistance. Bioactive peptides are short amino acid sequences that exert health benefits beyond their nutritional value. Egg white peptides and hydrolysates (EWH) have been shown to reduce blood pressure, inflammation and fat mass. Previously, feeding EWH for 6 weeks improved glucose and insulin tolerance in insulin resistant rats. Here, we aimed to identify EWH mechanism(s) of action related to glucose homeostasis. We 
hypothesize that EWH supplementation reduces local inflammation and increases insulin signaling in white adipose tissues in high fat diet (HFD)-induced insulin resistant rats. Sprague-Dawley rats were fed HFD for 6 weeks and then divided into 2 groups, HFD and HFD+4\% EWH for another 6 weeks. 10 min prior to euthanization insulin was injected in half of the animals in each group. WAT was tested for changes in insulin pathway activation (western blot), and inflammatory cytokines and adipokines secretion (ELISA). EWH enhanced Akt phosphorylation in WAT but no changes were seen regarding IR- $\beta$ phosphorylation or total GLUT4 amount. However, enhanced AS160 abundance in the EWH group suggested increased translocation of GLUT4 to plasma membrane. Local inflammatory cytokines, adiponectin and resistin extracted from WAT did not change after EWH feeding. In summary, EWH treatment improved WAT insulin signaling despite no improvement in local inflammatory state. Moreover, the effect on insulin signaling appeared to be downstream of insulin receptor activation (e.g. intracellularly).

\title{
P06 Effects of Ultrasound Processing on Rutin and its Derivatives
}

\section{Idaresit U. Ekaette, Marleny D. A. Saldaña}

Department of Agricultural, Food, and Nutritional Sciences, University of Alberta, Edmonton, AB, Canada

Ultrasonication is a green process widely utilized in the food industry for the mechanical disintegration of plant cell walls to enhance release of phenolic compounds. In this study, ultrasonication was used to determine its effect on a phenolic glycoside compound known as rutin. Rutin hydrate known for its pharmacological activities, was made into suspension of $20 \mathrm{~mL}, 1 \%(\mathrm{w} / \mathrm{v})$ with deionized water, $0.01 \mathrm{~g} / \mathrm{mL}$ citric acid and $0.01 \mathrm{~g} / \mathrm{mL}$ sodium chloride, then treated with an ultrasound tip of $20 \mathrm{~mm}$ diameter at $600 \mathrm{~W}$ (energy densities of 3.6, 9.0, 18.0, 27.0 and $36.0 \mathrm{~kJ} / \mathrm{mL}$ ) with temperature control $\left(47^{\circ} \mathrm{C}\right)$, and without temperature control $\left(86^{\circ} \mathrm{C}\right)$. Results showed significant increases in total phenolic content (TPC) of all ultrasound treated suspensions compared to untreated suspensions. The TPC increased from $0.27 \pm 0.01 \mathrm{mg}$ gallic acid equivalent $/ \mathrm{mg}$ rutin hydrate (untreated sample in deionized water) to $0.49 \pm 0.01 \mathrm{mg}$ gallic acid equivalent $/ \mathrm{mg}$ rutin hydrate (treated at $27 \mathrm{~kJ} / \mathrm{mL}$ and $47^{\circ} \mathrm{C}$ ). Similarly, the highest total flavonoid content (TFC) in deionized water $\left(0.44 \pm 0.01 \mathrm{mg}\right.$ catechin $/ \mathrm{mg}$ rutin hydrate) was obtained at 18 and $27 \mathrm{~kJ} / \mathrm{mL}$, and $47^{\circ} \mathrm{C}$. Metal (copper ion) chelating was observed only in $0.01 \mathrm{~g} / \mathrm{mL}$ citric acid treated samples, which increased significantly from $43 \%$ (untreated) to $76 \%$ (treated at $3.6 \mathrm{~kJ} / \mathrm{mL}$ and $86^{\circ} \mathrm{C}$ ). Antioxidant activities such as Ferric reducing antioxidant power (FRAP) and 2,2-diphenyl-1-picrylhydrazyl (DPPH) free radical scavenging were the lowest in $0.01 \mathrm{~g} / \mathrm{mL}$ sodium chloride treated samples. These results and the significant differences among individual samples showed that rutin might have hydrolyzed and modified to aglycone derivatives under ultrasonication treatment.

\section{P07 Analysis of Potential Synergistic Effects of Cannabis Extracts and Doxorubicin on the MCF-7/DOX Breast Cancer Cell Line}

\author{
Marina Galatonov ${ }^{1}$, Nina Takahashi ${ }^{1}$, Olga Kovalchuk ${ }^{1,2}$, Igor Kovalchuk ${ }^{1,2}$ \\ ${ }^{1}$ University of Lethbridge, Hepler Hall, Lethbridge, $A B$, Canada \\ ${ }^{2}$ Pathway RX,The University of Lethbridge, Lethbridge, $A B$, Canada
}

Doxorubicin is a commonly used chemotherapy drug in medicine. Despite being effective against tumors, it can produce various side effects in patients damaging normal cells and causing nausea, weakness and even cardiomyopathy. Additionally, some patients relapse with tumors that are resistant to chemotherapy drugs. The Cannabis sativa plant synthesizes various cannabinoids of which many have been shown to have anti-cancer properties. In this study, we analyze the effect of various cannabis extracts in combination with doxorubicin on the MCF-7/DOX breast cancer cell line which is resistant to doxorubicin. We hypothesize that cannabis extracts can potentiate the effect of chemotherapy drugs, thus, allowing a decrease in the amount of chemotherapy drug required to achieve the same cytostatic effect. Furthermore, cannabis extracts may aid in killing chemotherapy resistant cancer cell lines while having no effect on normal cells. MCF7/DOX cell cultures were treated with $1 \mu \mathrm{g} / \mathrm{mL}$ of doxorubicin in combination with various cannabis extracts for 48 hours or until cell death was observed. From microscopic analysis, it appears that doxorubicin in combination with extracts \#4, \#10 \#20, \#28 and \#41 at $0.05 \mu \mathrm{g} / \mu \mathrm{L}$ were most effective for inducing cell death in MCF-7/DOX cells in the span of $24 \mathrm{~h}$. In the future, we will evaluate the same cannabis extracts in combination with the cisplatin chemotherapy drug on the cisplatin resistant MCF-7/DPP breast cancer cell line. Western blots will be performed, and the expression of Cyclin D1 (proliferation marker), cleaved Parp and cleaved Casp3 (apoptosis markers) and H2AX (DNA damage marker) will be quantified to gain an insight to the pathways induced by the different combinations of compounds. 


\section{P08 Pressurized Gas eXpanded (PGX) Liquid Drying of Proteins \\ Yonas Gebrehiwot ${ }^{1}$, Ricardo Couto ${ }^{1}$, Bernhard Seifried ${ }^{2}$, Byron Yépez ${ }^{2}$, Paul Moquin², Feral Temelli \\ ${ }^{1}$ Department of Agricultural, Food and Nutritional Science, University of Alberta, Edmonton, AB, Canada \\ ${ }^{2}$ CEAPRO Inc., 7824 - 51 Avenue, Edmonton, $A B$, Canada}

In this work, Pressurized Gas eXpanded (PGX) liquid technology has been used to micronize and dry aqueous protein solutions as potential carrier for bioactive delivery systems. The effects of the initial concentration of soy protein isolate, aqueous solution flow rate and flow rate ratio of aqueous solution: EtOH:CO2 on the morphology, bulk density, specific surface area, particle size distribution, thermal stability and denaturation of the protein powder were investigated. The untapped bulk densities of the soy protein powders generated ranged from $0.0094 \pm 0.0002 \mathrm{~g} / \mathrm{mL}$ to $0.0221 \pm 0.0005 \mathrm{~g} / \mathrm{mL}$, which was more than an order of magnitude lower than that of the unprocessed protein at $0.2761 \pm 0.0003 \mathrm{~g} / \mathrm{mL}$. The PGX-dried samples exhibited much larger specific surface areas $\left(282 \pm 4.94 \mathrm{~m}^{2} / \mathrm{g}\right)$ compared to those of the unprocessed, spray, and freeze dried samples at $8.2 \mathrm{~m}^{2} / \mathrm{g}, 10.3 \mathrm{~m}^{2} / \mathrm{g}$ and $27.2 \mathrm{~m}^{2} / \mathrm{g}$, respectively. Larger specific surface areas were obtained for experimental conditions with low ethanol flow rate ratios and medium to low protein concentrations. Helium ion microscopy (HiM) images showed agglomerates of submicron particles were produced for all the experimental conditions tested. It was demonstrated that the PGX technology can be used to generate submicron protein particles having high specific surface area and low bulk densities without causing denaturation of proteins. The specific surface area of the particles generated is affected by the concentration of the feed protein solution, flow rate ratio, and $\mathrm{pH}$. The PGX technology is a suitable technique to generate protein powders having desirable characteristics with potential to be used as a carrier for bioactive delivery systems.

\section{P09 Production of Self-assembled Cellulose Nanofiber Hydrogels for Tissue Engineering}

\section{Raquel Razzera Huerta, Marleny D. A. Saldaña}

Department of Agricultural, Food and Nutritional Science, University of Alberta, Edmonton, AB, Canada

In the last decade, agricultural by-products like straw, have been a subject of intense study towards emerging biomaterial production. Cellulose nanofibers (CNFs) hydrogel is a promising biomaterial for medical and pharmaceutical applications due to its unique properties such as biodegradability, high specific strength and stiffness, high aspect ratio and surface area and light weight. In this study, the effect of ultrasonication at specific energy (SE) of 4-20 kJ/g under ambient conditions or cold bath was evaluated to disintegrate cellulose fibers from canola straw and obtain self-assembled CNFs hydrogels (1-2 wt.\%) with different residual lignin content. The heat induced by the acoustic cavitation under ambient conditions reached a maximum of $95^{\circ} \mathrm{C}$, which strongly affected the defibrillation process. Increasing the ultrasonication SE from 4 to $20 \mathrm{~kJ} / \mathrm{g}$, improved the dispersibility of the CNFs and increased the swelling capacity of the CNFs from 17 to $82 \mathrm{~g}$ water/g CNFs. Independent of the process temperature, the maximum yield of fibrillation (46 wt.\%) was obtained using ultrasonication at $20 \mathrm{~kJ} / \mathrm{g}$. Furthermore, regardless of the lignin content, the hydrogels obtained via ultrasonication exhibited a typical elastic gel-like behavior with maximum elasticity of $263 \mathrm{~Pa}$. The obtained CNFs hydrogels showed to be a promising source to be applied in biomedical area such as drug delivery, wound healing and scaffold for cell culture in tissue engineering applications.

\section{P10 In Vitro Study of Sulforaphane in Oxygen \& Glucose Deprived Brain Cells Zeenat Ladak, Elizabeth Garcia, Edward A. Armstrong, Jenny Yoon, Sujata Persad, Jerome Y. Yager \\ University of Alberta, Edmonton, AB, Canada}

Perinatal brain injury is responsible for a spectrum of neurodevelopmental disabilities. Current therapies target the injury after birth, 'rescuing' only a small percentage of injured newborns. Conventional pharmaceuticals can also cause harm to the developing brain. Thus, finding preventative therapeutics to protect the fetal brain is vital. Sulforaphane (SFN), the active ingredient of cruciferous vegetables, has shown promise for the injured fetal brain. Our objective is to evaluate the protective effect of SFN on brain cells exposed to oxygen and glucose deprivation (OGD). OGD simulates in vitro, the in vivo ischemic condition of placental insufficiency, a cause of perinatal brain injury. Our primary hypothesis is that SFN will prevent injury in neuronal cell cultures exposed to OGD. We developed a rodent newborn cell culture model of primary cortical neurons/astrocytes, which were exposed to 
OGD insult and $24 \mathrm{~h}$ of recovery with complete media. The effects of OGD were evaluated in the presence/absence of SFN at different doses to measure the efficacy of SFN. The purity of the cell cultures was evaluated by Western blot and immunofluorescence (IF) of cell-specific markers. Cell death was quantitated using viability analysis (IF/high content) and cytotoxicity using MTT assay. We determined the LD50 (duration of OGD required for 50\% cell death) as $2 \mathrm{~h}$ for neurons $(\mathrm{p}<0.001)$ and $4 \mathrm{~h}$ for astrocytes $(\mathrm{p}<0.002)$. We determined the dose of the protective effect of SFN for neurons was $2.5 \mathrm{uM}(\mathrm{p}<0.0001)$, and $5 \mathrm{uM}(\mathrm{p}<0.0001)$ for astrocytes. Statistical analysis using One Way ANOVA indicated that cell death was significantly reduced in neurons and astrocytes treated with SFN, suggesting that SFN could be a possible preventative for ischemic brain injury in the fetus.

\title{
P11 Bee Pollen Extracts Modulate Serum Metabolism in LPS-Induced Acute Lung Injury Mice with Anti-inflammatory Effects
}

Qiangqiang Li ${ }^{1}{ }^{2}$, Mirko Betti², Kai Wang ${ }^{1}$, Liming $\mathbf{W} \mathbf{u}^{1}$

${ }^{1}$ Institute of Apicultural Research, Chinese Academy of Agricultural Sciences, Beijing, China

${ }^{2}$ Faculty of Agricultural Life \& Environmental Sciences, University of Alberta, Edmonton, AB, Canada

Bee pollen (BP), as a natural treasure trove from the hive, has been highly promoted in a view of its various nutritional and health-beneficial properties, including boosting of the immune function and enhancing resistance to inflammatory damages. However, the lack of studies regarding the mechanisms of BP's action limits its further application. Here, we aimed to investigate both the in vitro and in vivo anti-inflammatory effects and metabolism-regulatory activities of BP from different botanical sources (i.e., Camellia sinensis L., BP-Cs; Nelumbo nucifera Gaertn., BP-Nn; Brassica campestris L., BP-Bc) based on molecular and metabolomic approaches. Firstly, the differences in the abundance of polyphenolic compounds among three types of BP extracts were compared using HPLC-DAD. Followed, the in vitro anti-inflammatory effects of three BP extracts were evaluated in lipopolysaccharide (LPS)-induced RAW 264.7 cells. It was found that BP-Cs extract with the most abundant polyphenols exerted the best anti-inflammatory effect via downregulating the expression of pro-inflammatory genes and blocking the activation of MAPK and NF-kB signaling pathways. Secondly, we addressed the in vivo anti-inflammatory activities of BP-Cs using LPS-challenged mice model with acute lung injury. An UPLC-Q-TOF/MS-based metabolomics approach was applied to analyze metabolites changes in the serum. It was found that the regular pretreatment with BP-Cs extract for one week alleviated the acute lung inflammation, which was potentially associated with the glycerophospholipid metabolism regulated by BP-Cs extract in LPS-challenged mice. These in vitro and in vivo findings might extend the future utilization of bee pollen in functional food and pharmaceutical industries.

\section{P12 Pressurized Gas eXpanded (PGX) Liquid Drying of Sodium Alginate and Loading with Coenzyme Q10}

\author{
Zixiang Liu' ${ }^{1}$, Ricardo Couto', Bernhard Seifried ${ }^{2}$, Byron Yépez ${ }^{2}$, Paul Moquin², Feral Temelli \\ ${ }^{1}$ University of Alberta, Edmonton, $A B$, Canada \\ ${ }^{2}$ CEAPRO Inc., Edmonton, $A B$, Canada
}

The Pressurized Gas eXpanded (PGX) liquid drying technology is able to dry high molecular weight biopolymers from aqueous solutions. This technology only uses food grade anhydrous ethanol $(\mathrm{EtOH})$ and carbon dioxide $\left(\mathrm{CO}_{2}\right)$ mixture to perform drying at moderate conditions $\left(40^{\circ} \mathrm{C}\right.$ and $\left.100 \mathrm{bar}\right)$. Sodium alginate $(\mathrm{SA})$ is a polysaccharide extracted from brown algae, which has a high molecular weight ranging from 80,000 to $200,000 \mathrm{~g} / \mathrm{mol}$, and is difficult to dry using traditional drying methods. Coenzyme Q10 (CoQ10) is a lipid-soluble antioxidant, mainly present in the mitochondria of eukaryotic cells, which can benefit human cardiovascular health. However, its applications in aqueous systems are limited because of its hydrophobic nature. The objective of this study was to investigate the effect of processing parameters on the physicochemical properties of PGX-dried SA, and load it with CoQ10 as a delivery system. Different processing parameters, including the concentration of SA in the aqueous solution, flow rate of $\mathrm{EtOH}$, and weight ratio of $\mathrm{CO}_{2}$ : $\mathrm{EtOH}$ :aqueous solution were tested. CoQ10 loading amount and dispersibility in water were also determined. The optimal processing condition was obtained by using 1.0\% w/w SA solution, $15 \mathrm{~g} / \mathrm{min}$ EtOH flow rate, with 5:15:4 weight ratio, which resulted in a very high surface area $\left(164.5 \mathrm{~m}^{2} / \mathrm{g}\right)$ of dried SA. The morphology of PGX-processed SA consisted of nanofibrils, which could not be obtained by spray drying or freeze drying. Adsorptive precipitation of CoQ10 on PGX-dried SA using supercritical $\mathrm{CO}_{2}$ resulted in homogeneous loading of CoQ10 on SA particles, which could be dispersed uniformly in water. The findings demonstrate the potential for broad applications of CoQ10 in beverage and cosmetic products. 


\title{
P13 Evaluation of Concentrated Caffeine in Supplemented Foods and Natural Health Products
}

\section{Kasey P. Mohan, Bradley J. Lampe}

NSF International, Ann Arbor, MI, United States

Despite their ubiquity in the marketplace, the safety of functional foods and Natural Health Products/supplements containing concentrated caffeine has been widely scrutinized, due in part to their potential to contain relatively large doses of caffeine in a capsule or "shot" form. A literature review was conducted to determine whether: (a) the ingested concentration of caffeine and (b) the duration over which the caffeine dose is consumed (i.e. consumption time) impact toxicokinetic parameters in humans. Five human studies were located in which cardiovascular and/or pharmacological endpoints were measured following the administration of varying concentrations and volumes of caffeine. These endpoints included: QT interval, heart rate, blood pressure, maximum serum concentration (Cmax), time at which this concentration was achieved (Tmax), and clearance. All five studies indicated that the toxicokinetic parameters of caffeine are not dependent on its concentration in a consumed beverage. Furthermore, a study in 24 human volunteers who consumed either coffee or an energy drink (each dose contained approximately $0.04 \%$ caffeine) over durations of $2 \mathrm{~min}$ or 20 min concluded that the consumption time did not significantly affect the Cmax, Tmax, or clearance of caffeine. Although the data are limited, the results of these studies indicate that there is insufficient evidence to suggest that concentrated caffeine sources pose any additional hazard in comparison with conventional caffeinated energy drinks. Future research in this area would be beneficial to provide a more robust dataset to substantiate this conclusion.

\section{P14 Sesquiterpene Lactones: Natural Products Found in Prairie Plants that Inhibit Cells in Mitosis}

\author{
Layla A. Molina', Alessandra Bosco', Chad Beck', Raymond Andersen², Roy M. Golsteyn' \\ ${ }^{1}$ Natural Product Laboratory, University of Lethbridge, Lethbridge, AB, Canada \\ 2Department of Earth, Ocean and Atmospheric Sciences, University of British Columbia, Vancouver, BC, Canada
}

In the Prairie to Pharmacy Program we investigate endemic plants from the Prairie ecological region for natural products that have biological activities of interest. We had previously reported the isolation and characterization of the sesquiterpene lactone (SL), Pulchelloid A, from the plant Gaillardia aristata, which arrested cancer cells in mitosis. SLs contain a 15-carbon backbone, including a lactone group, and are secondary metabolites of many plant families, especially Asteraceae. Although more than 5000 SLs have been described in the literature, only 10 have been reported to arrest the cell cycle. We have identified a second SL, Hymenoratin, by biology-guided fractionation from the plant Hymenoxys richardsonii. Hymenoratin arrests in mitosis about $30 \%$ of the population of HT29 cells, measured by cell rounding, presence of phospho-histone $\mathrm{H} 3$, spindle formation, and accumulation of cells in G2/M phases. In addition, arrested cells accumulate the proteins Cyclin B, Plk1, and an active Cdk1 complex. Strikingly, treated cells undergo a failure in chromosome congression, distortion of the mitotic spindle, and acquire damaged DNA signals but only in mitotic chromosomes. To date, we have identified 6 Asteraceae species extracts with cell rounding activity out of 16 species tested, revealing that the anti-mitotic activities are not universal. Recently, we detected an anti-mitotic activity similar to that of Asteraceae family members in extracts prepared from the Prairie plant Primula conjugens, from Primulaceae, which has not been previously reported as a source of anti-mitotic compounds. Canadian Prairie plants are proving to be a rich source of anti-mitotic natural products for scientific and anti-cancer investigation.

\section{P15 Natural Health Product Researchers' Attitudes Towards Open Access Publishing and a New Field-specific Journal: A Research Protocol \\ Jeremy Y. Ng ${ }^{1,2}$, Kieran Cooley 2,3,4,5, Pierre S. Haddad 2,6 , Fred Ashbury ${ }^{2,7}$ \\ ${ }^{1}$ Department of Health Research Methods, Evidence, and Impact, Faculty of Health Sciences, McMaster University, Hamilton, ON, Canada \\ ${ }^{2}$ NHP Publications, Toronto, ON, Canada \\ ${ }^{3}$ Australian Research Center in Complementary and Integrative Medicine, University of Technology, Ultimo, Australia \\ ${ }^{4}$ Pacific College of Oriental Medicine, San Diego, CA, United States \\ ${ }^{5}$ Department of Research, Canadian College of Naturopathic Medicine, Toronto, ON, Canada \\ ${ }^{6}$ Department of Pharmacology and Physiology, Faculty of Medicine, University of Montreal, Montreal, QC, Canada \\ 7 Institute of Health Policy, Management and Evaluation, University of Toronto, Toronto, ON, Canada}

Open access publishing was proposed as a solution to the traditional subscription-based model, since it allows authors to retain copyright of their publication and to make it freely and publicly available following a one-time 
article processing fee. While some studies have explored researchers' attitudes towards open access publishing, to our knowledge our study is the first to be conducted to help create and improve a new field-specific open access scholarly journal. We will conduct an international survey of natural health product (NHP) researchers. Potential survey respondents will be sourced from information publically available on the internet. Survey questions will be designed to gauge NHP researchers' understanding of and experience with open access publishing, as well as needs, desires and capacities related to making their research work publicly available. Data will be collected online and transferred to a database. Key patterns and issues concerning knowledge and attitudes regarding open-access publishing, including opportunities to improve the experience and its impact, will be identified using descriptive analytics and thematic analysis. Results will be used to implement optimal changes and heighten NHP researchers' experience in publishing in an open-access journal regarding their primary research interests, namely the Journal of Natural Health Product Research. This approach presents a unique opportunity for NHP researchers to tailor a new field-specific, academic research journal to their needs and to disseminate their research in a unique central publication designed to connect researchers internationally.

\title{
P16 Disclosure of Natural Health Product Use among Naturopathic Clinic Attendees: A Cross-Sectional Study
}

\section{Jeremy Y. Ng${ }^{1}$, Anna Garber ${ }^{2}$, Michelle Luong ${ }^{1}$, Kieran Cooley ${ }^{2,3,4}$, Jason W. Busse ${ }^{1}$}

${ }^{1}$ Department of Health Research Methods, Evidence, and Impact, Faculty of Health Sciences, McMaster University, Hamilton, ON, Canada ${ }^{2}$ Department of Research, Canadian College of Naturopathic Medicine, Toronto, ON, Canada

${ }^{3}$ Australian Research Center in Complementary and Integrative Medicine, University of Technology, Ultimo, Australia

${ }^{4}$ Pacific College of Oriental Medicine, San Diego, CA, United States

A 2003 survey of 198 adult patients (88\% response rate) attending the Robert Schad Naturopathic Clinic (teaching clinic of the Canadian College of Naturopathic Medicine) in Toronto, Canada found that $93 \%$ used natural health products (NHPs), of which $42 \%$ did not disclose use to their primary care physician. Lack of disclosure of NHP use makes recognizing and reporting adverse events and drug interactions difficult for clinicians and results in missed opportunities to discuss and understand the health concerns of patients. We repeated the same survey in 2018/2019, at the same clinic, to explore if rates of disclosure had improved over the intervening 15 years. Of 1112 adult naturopathic patients attending the clinic during 2 randomly selected 10-day intervals, 277 (25\%) completed the survey. Most respondents were female (76\%), and 114 (41\%) of respondents did not discuss NHP use with their medical doctor, suggesting that disclosure rates have not changed in 15 years. Participants reported taking the following: vitamins and minerals $(87 \% \mathrm{n}=240)$, herbs $(59 \%, \mathrm{n}=164)$, probiotics $(51 \%, \mathrm{n}=141)$, homeopathic remedies $(25 \%$, $\mathrm{n}=69)$, traditional Chinese medicines $(15 \%, \mathrm{n}=42)$, and medical cannabis $(9 \%, \mathrm{n}=25)$. While most naturopathic patients we surveyed reported use of a wide range of NHPs, many do not disclose such use with their medical doctor. Low response rate, setting, sampling, and nature of participant recall all limit the generalizability and accuracy of our results. Given the persistence of non-disclosure of NHP use by patients, healthcare providers should have an open conversation about NHP use, particularly those patients who attend for naturopathic care, in order to optimize care and reduce the risk of natural product-drug interactions and minimize medical error.

\section{P17 In Vitro Cytotoxic Activity of Ethanolic Extract of Dracaena Cochinchinensis Against Liver and Cholangiocarcinoma Cancer Cell Lines and its Anti-inflammatory Effect}

\author{
Nuntika Prommee ${ }^{1}$, Arunporn Itharat ${ }^{2}$, Sumalee Panthong ${ }^{2}$, Sunita Makchuchit ${ }^{2}$, Buncha Ooraikul ${ }^{3}$ \\ 'Student of Doctor of Philosophy (Applied Thai Traditional Medicine), Department of Applied Thai Traditional Medicine, Faculty of Medicine, \\ Thammasat University (Rangsit campus), Klongluang, Pathumthani, Thailand \\ ${ }^{2}$ Department of Applied Thai Traditional Medicine and Center of Excellence in Applied Thai Traditional Medicine Research (CEATMR), Faculty of \\ Medicine, Thammasat University (Rangsit campus), Klongluang, Pathumthani, Thailand \\ ${ }^{3}$ Professor Emeritus, Agricultural Life and Environmental Sciences, Agricultural Food and Nutritional Science, University of Alberta, Edmonton, \\ $A B$, Canada
}

Dracaena cochinchinensis (DC) heartwood is used in Asia as a folk medicine remedy, for antipyretic, circulatory stimulation, and anti-inflammatory properties. Some Thai traditional practitioners use DC to treat cancer. This study is to investigate the cytotoxic activity of 95\% ethanolic extract of DC (DCE95) against two types of human cancer cell lines: hepatocarcinoma cell line (Hep G2) and cholangiocarcinoma cell line (KKU-M156) and 
compared with one type of normal cell such as human keratinocyte cell line (HaCat) by using Sulforhodamine B (SRB) assay. Anti-inflammatory effect of its extract was tested by using lipopolysaccharide (LPS)-induced nitric oxide (NO) and determination by Griess reaction. Prostaglandin E2 (PGE2) was determined by EnzymeLinked Immunosorbent Assay (ELISA) test kit in murine macrophage (RAW 264.7) cell lines. Results were found that DCE95 exhibited high cytotoxic activity against Hep G2 and KKU-M156 cell lines with IC50 values at $7.723 \pm 1.876$ and $5.272 \pm 5.017 \mu \mathrm{g} / \mathrm{mL}$, respectively. DCE95 showed moderate cytotoxic activity against normal cell line (HaCat) with IC50 value at $48.097 \pm 0.807 \mu \mathrm{g} / \mathrm{mL}$. For the anti-inflammatory effect on NO and PGE2 inhibition, DCE95 showed higher anti-inflammatory effects than standard Acetaminophen with IC50 values at $33.825 \pm 0.564$ and $3.063 \pm 0.256 \mu \mathrm{g} / \mathrm{mL}$, respectively and Acetaminophen showed NO and PGE2 inhibitory effects with IC50 values at $>100$ and $6.110 \pm 0.661 \mu \mathrm{g} / \mathrm{mL}$, respectively. These findings suggest that DCE95 should be continuously studied in vivo model and clinical trial for the treatment of liver and cholangiocarcinoma cancers.

\section{P18 Determination of Ethanol Content in Kombucha Using Headspace Gas Chromatography with Mass Spectrometry Detection: Single-laboratory Validation}

\section{Jake Robertson', Hong Sy1,2, Michael Chan², Paula N. Brown²}

${ }^{1}$ British Columbia Institute of Technology, Department of Food Technology, Burnaby, BC, Canada

${ }^{2}$ British Columbia Institute of Technology, Centre for Applied Research \& Innovation, Burnaby, BC, Canada

Kombucha is a fermented tea beverage thought to have originated in China approximately 2000 years ago, which has recently gained popularity in the North American marketplace. Comprised of tea, sugar and a symbiotic culture of bacteria and yeast, commonly known as SCOBY. Although it can include other ingredients such as flavourings or acids, the alcohol content must be less than $0.5 \% \mathrm{ABV}$ (alcohol by volume), as it is marketed as a non-alcoholic beverage. Given the legal requirement for maintaining less than $0.5 \% \mathrm{ABV}$, there is a need for a reliable and accurate method to determine alcohol content in this complex matrix. A headspace gas chromatographic method with mass spectral detection was developed and evaluated for performance characteristics, as per AOAC International guidelines, including selectivity, accuracy, repeatability, and limits of detection. With a linear dynamic range of $0.05-4.05 \% \mathrm{ABV}$, the method was found to be fit for the purpose of determining alcohol content in Kombucha products with \%RSDr values $\leq 4.9 \%$ and a limit of detection of $0.002 \%$ ABV. Analysis of commercial products employing this method demonstrated 3 of 4 products contained ethanol above the legal limit of $0.5 \% \mathrm{ABV}$, posing a public health risk to consumers.

\section{P19 Bioactive Pectin Films Loaded with Encapsulated Vitamins (D3/K2) to Relief Baby Teething}

\section{Carla Valdivieso Ramirez, Marleny D. A. Saldaña}

Department of Agricultural, Food, and Nutritional Sciences, University of Alberta, Edmonton, AB, Canada

Studies have found that deficiency of vitamins D3/K2 in infants is linked to early tooth decay, narrow jaws and, increased incidence of rickets and pneumonia, particularly, in countries that are far from the tropics. In addition, teething process is known to be challenging for babies as it is associated to inflamed gums, drooling, lack of appetite and, even serious infections due to babies' tendency to chew on hands and things. Current teething treatments, however, include pain relievers and plastic teethers that are cause of either stomach irritation or poisoning due to chemicals released from plastics. Therefore, the aim of this study was to obtain a natural and safe product that could sooth baby's teething and, at the same time, support healthy primary teeth growth. Therefore, a chewable, anti-inflammatory and anesthetic pectin-based film loaded with encapsulated vitamins D3/K2 and essential oils (EOs) was produced. A three-stage green process was carried out, which involved: i) subcritical water (scW) hydrolysis of pectin, ii) encapsulation of vitamins (D3/K2) and essential oils (chamomile/clove) using a combined emulsification, electric vibration and ionotropic-gelation method and, iii) polymer-casting for film formation. Results showed that pectic hydrolysis was favored at $140^{\circ} \mathrm{C} / 50 \mathrm{bar} / 30 \mathrm{~min}$ as the molecular size reduction was $80 \%$. However, pectin hydrolysates obtained at $100^{\circ} \mathrm{C}$ let to stable pectin-base microcapsules loaded with either vitamins $\mathrm{D} 3 / \mathrm{K} 2$ or EOs. Finally, scW technology combine with polymer-casting proved to be a feasible process to obtain bioactive, edible and flexible pectin films for potential baby oral care. 


\section{P20 Anti-Neuroinflammatory and Neuroprotective Effects of Combined Treatment with Ubisol-Q10 and Ashwagandha Root Extract in a Paraquat Induced Rat Model of Parkinson's Disease

\author{
Caleb Vegh', Iva Okaj', Darcy Wear', Rachel Huggard", Lauren Culmone', Sezen Eren², Chris Nguyen', \\ Jerome Cohen², Siyaram Pandey ${ }^{1}$ \\ ${ }^{1}$ Department of Chemistry and Biochemistry, University of Windsor, Windsor, ON, Canada \\ ${ }^{2}$ Department of Psychology, University of Windsor, Windsor, ON, Canada
}

Parkinson's disease (PD) is characterized by neuron loss in the substatia nigra (SN) pars compacta region of the brain resulting in loss of movement coordination which progresses to morbidity and mortality. There is no treatment to stop the progression of neurodegeneration and current therapies such as dopamine supplements or deep brain stimulation only provide symptomatic relief. Previously we have shown Ubisol-Q10, a water-soluble formulation of coenzyme-Q10 shows near-complete protection in-vitro and in-vivo. Similarly, ethanolic root extract of ashwagandha (ASH) showed neuroprotection in a mouse model of PD. Here we combine ASH with Ubisol-Q10 in a paraquat induced PD rat model. Using a multidisciplinary approach, we demonstrated that paraquat treated rats given Ubisol-Q10, ASH or a combination of both (protected groups) in drinking water had reduced motor impairment compared to unprotected rats. Immunohistochemical analysis indicated significant protection of SN neurons, and enhanced activation of pro-survival astroglia and inhibition of pro-inflammatory microglia. In addition, this regiment caused activation of autophagy which may be responsible healthier neurons. Upregulation of both glial and brain derived neurotrophic factors was seen in protected groups indicating support of survival mechanism of neurons. Furthermore, both of these materials are in the category of nutritional supplements and can be given to patients over a long period of time without any adverse side effects. Based on these interesting observations, the combined treatment with Ubisol-Q10 and ASH could be an effective strategy to halt the progression of $\mathrm{PD}$ compared to using either nutraceutical alone.

\section{P21 Extraction of Coenzyme Q10 from Chicken Liver Using Supercritical Carbon Dioxide David Villanueva-Bermejo, Feral Temelli}

Department of Agricultural, Food and Nutritional Science, University of Alberta, Edmonton, AB, Canada

Coenzyme Q10 (CoQ10) is a benzoquinone, which has interesting health benefits, such as antioxidant, anti-cancer and anti-diabetic properties. CoQ10 is ubiquitously distributed in natural sources, but only some meats, fish and oils contain substantial amounts. Non-polar petroleum-based organic solvents are efficient for its extraction, but they are toxic and harmful to the environment. Moreover, CoQ10 is sensitive to oxygen and heat, so its extraction is challenging. Supercritical carbon dioxide $\left(\mathrm{SCCO}_{2}\right)$ is proposed as an alternative. $\mathrm{SCCO}_{2}$ is green, non-toxic, shows a high solvation power and allows performing extractions at low temperature in the absence of oxygen, avoiding the degradation of sensitive compounds. A potential and unexplored source of CoQ10 is chicken liver, a food industry byproduct which constitutes an environmental problem. In the present study, the extraction of CoQ10 from chicken liver using $\mathrm{SCCO}_{2}$ was explored. The effects of pressure (15-45 MPa), temperature $\left(40\right.$ and $\left.60^{\circ} \mathrm{C}\right)$ and the use of ethanol as a modifier were studied. Oil extraction yields around $20 \%$ were obtained, which were slightly higher with ethanol addition ( $25 \%$ at $45 \mathrm{MPa}, 40^{\circ} \mathrm{C}$ and $10 \%$ ethanol). The largest concentrations of CoQ10 in the oil were obtained at the end of the extraction period $\left(>7 \mathrm{mg} / \mathrm{g}\right.$ at $15 \mathrm{MPa}$ and $\left.40^{\circ} \mathrm{C}\right)$, which is driven by the diffusion of CoQ10 in the solid material. Nevertheless, the highest solubility of $\mathrm{CoQ} 10$ and oil in $\mathrm{CO}_{2}$ was achieved during the constant extraction rate period, which is driven by the solubility of the target compound in the solvent. $\mathrm{SCCO}_{2}$ extraction of CoQ10 from chicken liver shows great potential for the recovery of a high value ingredient from a byproduct.

\section{P22 Autophagy Resumption by Ubisol-Q10 Ameliorates Alzheimer's Disease Symptoms and Pathologies \\ Darcy Wear', Caleb Vegh', Simon Pupulin'1, Rachel Huggard', Lauren Culmone', Iva Okaj', Eren Sezen ${ }^{2}$, Jerome Cohen'², Siyaram Pandey ${ }^{1}$ \\ 'Department of Chemistry and Biochemistry, University of Windsor, Windsor, ON, Canada \\ ${ }^{2}$ Department of Psychology, University of Windsor, Windsor, ON, Canada}

Alzheimer's disease (AD) is the most common neurodegenerative disorder, affecting 48 million individuals worldwide, and is associated with loss of memory, amyloid-beta plaque buildup and neurofibrillary tangles. These features 
might be a result of neuronal cell death in the cerebral cortex and hippocampal regions of the brain. The AD pathologies can be attributed to a variety of biochemical consequences including inflammation, mitochondrial dysfunction, increased oxidative stress, and autophagy inhibition. Current treatments focus only on symptomatic relief instead of inhibiting AD's progression. Previous in-vitro experiments have shown that a water-soluble formulation of coenzyme-Q10, Ubisol-Q10, can stabilize the mitochondria, prevent oxidative stress and inhibit premature senescence in fibroblasts of AD patients. Since autophagy plays a critical role in maintenance and survival of neurons, we hypothesized that Ubisol-Q10 treatment could result in resumption of autophagy. Indeed, we observed induction of autophagy by Ubisol-Q10 treatment in AD fibroblasts as well as in the brains of transgenic AD mice. We found increased expression of autophagy related genes following Ubisol-Q10 treatment. These results were confirmed at the protein level by immunohistochemistry and Western blotting. Additionally, Ubisol-Q10 was shown to inhibit amyloid-beta plaque formation in transgenic $\mathrm{AD}$ mouse brains while improving their long term and spatial memory. These results indicate that autophagy seems to play a key role in neuronal health, and treatment with Ubisol-Q10 may block progression of Alzheimer's Disease.

\section{Conflicts of Interest}

The author(s) declare that they have no conflicts of interest.

\section{Authors' Contributions}

PNB: made the call for abstracts on behalf of the NHPRS, reviewed the abstracts for acceptance as oral and poster presentation and contributed to the copyediting and review of the publication.

(D) https://orcid.org/0000-0003-4886-7001

RMG: made the call for abstracts on behalf of the NHPRS, reviewed the abstracts for acceptance as oral and poster presentation and contributed to the copyediting and review of the publication.

(D) https://orcid.org/0000-0002-0251-7025

\section{Article Information}

Managing Editor: Kieran Cooley, Pierre Haddad

Peer Reviewers: This article was peer-reviewed by the NHPRS conference planning committee.

Article Dates: Published May 2619

\section{Citation}

Please cite this article as follows:

Brown PN, Golsteyn RM. Growing NHPs for the Future: The 16th Annual Natural Health Products Research Conference and Tradeshow. Journal of Natural Health Product Research. 2019 May 26: Vol1(1). https://jnhpresearch.com/index.php/jnhpr/article/view/5

DOI Link: https://doi.org/10.33211/jnhpr.5

\section{Copyright}

(c) Paula N. Brown, Roy M. Golsteyn. (2019). Published first in the Journal of Natural Health Product Research. This is an open access article distributed under the terms of the Creative Commons Attribution License (https:// creativecommons.org/licenses/by/4.0/), which permits unrestricted use, distribution, and reproduction in any medium, provided the original work, first published in the Journal of Natural Health Product Research, an NHP Publications journal, is properly cited. The complete bibliographic information, a link to the original publication on https://www.jnhpresearch.com, as well as this copyright and license information must be included. 
Journal of Natural Health Product Research NTIT TT TRTTRATTONTS

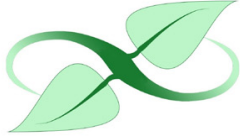

Canadà̀

Does the safety, efficacy, and quality of natural health products matter to YOU? Submit your research article to the Journal of Natural Health Product Research!

Pre-submission inquiries? Send us an email at editorial.office@jnhpresearch.com Facebook, Twitter and LinkedIn: @NHPPublications 